Research Article

\title{
Boundedness of Singular Integral Operators with Operator-Valued Kernels and Maximal Regularity of Sectorial Operators in Variable Lebesgue Spaces
}

\author{
Qinghua Zhang $\mathbb{D}^{\text {D }}$, Yueping Zhu, and Feng Wang \\ School of Sciences, Nantong University, Nantong, China \\ Correspondence should be addressed to Qinghua Zhang; zhangqh1971@126.com
}

Received 29 September 2019; Accepted 11 November 2019; Published 10 March 2020

Academic Editor: Yongsheng S. Han

Copyright (c) 2020 Qinghua Zhang et al. This is an open access article distributed under the Creative Commons Attribution License, which permits unrestricted use, distribution, and reproduction in any medium, provided the original work is properly cited.

\begin{abstract}
This paper is devoted to the maximal regularity of sectorial operators in Lebesgue spaces $L^{p(\cdot)}$ with a variable exponent. By extending the boundedness of singular integral operators in variable Lebesgue spaces from scalar type to abstract-valued type, the maximal $L^{p^{(\cdot)}}$-regularity of sectorial operators is established. This paper also investigates the trace of the maximal regularity space $\mathbb{E}_{0}^{1, p(\cdot)}(I)$, together with the imbedding property of $\mathbb{E}_{0}^{1, p(\cdot)}(I)$ into the range-varying function space $C^{-}\left(I, X_{1-1 / p(\cdot), p(\cdot)}\right)$. Finally, a type of semilinear evolution equations with domain-varying nonlinearities is taken into account.
\end{abstract}

\section{Introduction}

Maximal $L^{p}$-regularity of sectorial operators is an important theory, which brings a powerful tool in investigating the evolution equations in $L^{p}$-spaces. Let $X$ be a Banach space and $A$ be a closed operator defined in $X$ with the dense domain $\mathscr{D}(A)$ and dense range $R(A)$, and let $X_{1}=\mathscr{D}(A)$ endowed with the graph norm. $A$ is called a sectorial operator, if there are constants $M_{0}>0$ and $0<\omega<\pi / 2$, such that the sector

$$
\Sigma_{\omega}=\{\lambda \in|\mathbb{C}\{0\}:| \arg \lambda \mid \leq \pi-\omega\},
$$

is contained in $\varrho(-A)$, and the inequality

$$
\left\|(\lambda I+A)^{-1}\right\| \leq \frac{M}{|\lambda|},
$$

holds for all $\lambda \in \Sigma_{\omega}$. Recall that for a sectorial operator $A$, its negative $-A$ generates an analytic $C_{0}$-semigroup $e^{-t A}$ (refer to [1], Section 2.5).

Let $I=[0, b]$ with $0<b<\infty$ or $I=[0, \infty)$, and consider the abstract differential equation

$$
u^{\prime}(t)+A u(t)=f(t), \quad 0<t \leq b .
$$

We say that $A$ satisfies the maximal $L^{p}$-regularity on $I$, or $A \in \mathscr{M} \mathscr{R}_{p}(I)$ in symbol, if for all $f \in L^{p}(I, X)$, there is a unique solution $u \in W^{1, p}(I, X) \cup L^{1, p}\left(I, X_{1}\right)$ of equation (3) with the initial value $u(0)=0$. Using the interpolation method for the convolution operators with singular kernels, we know that (see $[2,3]$, etc.) if $A \in \mathscr{M} \mathscr{R}_{q}(I)$ for some $1<q<\infty$, then $A \in \mathscr{M} \mathscr{R}_{p}(I)$ for all $1<p<\infty$.

In $[4,5]$, the authors gave a general introduction on the $L^{p}$-regularity of sectorial operators and [6-9] investigated the maximal $L^{p}$-regularity of the second order elliptic and Stokes operators, made some $L^{p}-$ or $L^{p}-L^{q}$-estimates for the parabolic evolution and nonstationary Navier-Stokes equations. Maximal regularity of sectorial operators in weighted $L^{p}$-spaces was established in [10] and applied in quasilinear equations in $[11,12]$. During the same period, Chill and Fiorenza [13] dealt with the maximal regularity of sectorial operators in Orlicz spaces of rearrangement invariant Banach functions.

In some concrete situations, the nonlinear term $f$ attached to (3) may be lying in $L^{p(\cdot)}(I, X)$, so it is natural to consider the maximal regularity in such spaces. Since $p(\cdot)$ is a variable exponent, the interpolation method used in $[2,3]$ is not suitable anymore. Because of lacking of translation 
invariance, space $L^{p(\cdot)}(I, X)$ is not arrangement invariant, hence tools developed in [13] are not applicable directly yet. In order to establish the maximal $L^{p(\cdot)}$-regularity of $A$, a recently developed method for the maximal operator and singular integral operators can be employed. This method is associated with the maximal operator $M$, the sharp maximal operator $M^{\#}$ and the singular integral operator $T$ attached with $A$ in $L^{p}$-spaces with variable exponents (refer to $[14,15])$. By employing this method, with the aid of the estimate obtained in [13], in this paper, we will prove that if $A \in \mathscr{M} \mathscr{R}_{q}(I)$ for some $1<q<\infty$, then $A \in \mathscr{M} \mathscr{R}_{p(\cdot)}(I)$ for all log-Hölder continuous exponents $p(\cdot)$ with $1<p^{-}<p^{+}<\infty$, where $p^{+}$and $p^{-}$denote the supremum and infimum of $p(\cdot)$ on the interval $I$, respectively.

In order to apply the maximal $L^{p(\cdot)}$-regularity theory to the quasilinear evolution equations, in this paper, we also make some investigations on the trace of the maximal regularity space $W^{1, p(\cdot)}(I, X) \cap L^{1, p(\cdot)}\left(I, X_{1}\right)$. As we know that, for all subintervals $J$ of $I, W^{1, p_{I}^{-}}(J, X) \cap L^{1, p_{I}^{-}}\left(J, X_{1}\right)$ can be imbedded into the space $C\left(J, X_{1-1 / p_{J}^{-}, p_{J}^{-}}\right)($refer to $[5,10])$, where $p_{J}^{-}=\inf _{t \in J} p(t)$, and $X_{1-1 / p_{J}^{-}, p_{J}^{-}}=\left(X, X_{1}\right)_{1-1 / p_{J}^{-}, p_{J}^{-}}$is the real interpolation space between $X$ and $X_{1}$, a question arises naturally, that is, for arbitrary $t \in I$, whether or not the trace space of $W^{1, p(\cdot)}(I, X) \cap L^{1, p(\cdot)}\left(I, X_{1}\right)$ is $X_{1-1 / p(t), p(t)}$ exactly. This question was raised in [16] and has had not an answer until now. The main obstacle is that the imbedding bounds of $W^{1, p_{J}^{-}}(J, X) \cup L^{1, p_{J}^{-}}\left(J, X_{1}\right) \hookrightarrow C\left(J, X_{1-1 / p_{J}^{-}, p_{J}^{-}}\right)$depend on the length of $J$, and it could not be controlled as the interval $J$ shrinks to the point $t$. Here, by using the properties of the log-Hölder function, together with the theory of range-varying function spaces developed in $[16,17]$, we give this question an affirmative answer. We will show that, in case that $-A$ generates a exponentially decaying semigroup and $p(\cdot)$ is a log-Hölder function, then the homogeneous maximal regularity space $W_{0}^{1, p(\cdot)}(I, X) \cap L^{1, p(\cdot)}\left(I, X_{1}\right)$ can be imbedded in $C^{-}\left(J, X_{1-1 / p(\cdot), p(.)}\right)$, a range-varying function space established on the regular Banach space net $\left\{X_{\alpha, p(\alpha)}: \alpha \in[0,1]\right\}$. This gives an affirmative answer to the question about the trace of the homogeneous maximal regularity space.

This paper is organized as follows. As preliminaries, in this and the next sections, we make a brief review on the maximal $L^{p}$-regularity of sectorial operators and the $X_{\theta(\cdot)}$-valued function spaces. In Section 3, the main results on singular integral operators with operator-valued kernels with application to maximal regularity in $L^{p(\cdot)}(I, X)$ and time-varying trace of the maximal regularity space are derived. All the results will be applied to a semilinear evolution equation with the time-dependent nonlinearity at the end of the paper. This example implies the wide application of our work in the study of parabolic partial differential equations with nonstandard growth.

\section{Preliminaries}

Given a Banach space $X$ and a sectorial operator $A$ which is densely defined in $X$. Let $X_{1}=\mathscr{D}(A)$ endowed with the graph norm as above, and let $I=[0, b]$ or $I=[0, \infty)$.
Given $1<q<\infty$, define the maximal regularity space

$$
\mathbb{E}^{1, q}(I)=W^{1, q}(I, X) \cap L^{q}\left(I, X_{1}\right),
$$

endowed with the norm $\|u\|_{\mathbb{E}^{1, q}(I)}=\|u\|_{W^{1, q(I, X)}}+\|u\|_{L^{q}\left(I, X_{1}\right)}$ and the homogeneous subspace

$$
\mathbb{E}_{0}^{1, q}(I)=\left\{u \in \mathbb{E}^{1, q}(I), u(0)=0\right\} .
$$

Under present situations, $\mathbb{E}^{1, q}(I) \hookrightarrow C\left(I, X_{1-1 / q, q}\right)$ for $I=$ $[0, \infty)$ and $\mathbb{E}_{0}^{1, q}(I) \hookrightarrow C\left(I, X_{1-1 / q, q}\right)$ for $I=[0, b]$ with the imbedding bounds independent of $b>0$ (refer to [18], Section 3.4.10).

By the inverse operator theorem of the closed operators, we can assert that, if $A \in \mathscr{M}_{\mathscr{R}_{q}}(I)$, then there is a constant $C_{q}>0$ such that

$$
\|u\|_{\mathbb{E}^{1, q}(I)} \leq C_{q}\|f\|_{L^{q}(I, X)},
$$

where $f \in L^{q}(I, X)$ and $u \in \mathbb{E}_{0}^{1, q}(I)$ is the solution of equation (3). Furthermore, if $A \in \mathscr{M} \mathscr{R}_{q}\left(\mathbb{R}^{+}\right)$, then $C_{q}$ is independent of the length of $I$, and $-A$ generates an exponentially decaying analytic semigroups $e^{-t A}$, i.e., there are constants $M_{0} \geq 1$ and $\omega>0$ such that

$$
\max \left\{\left\|e^{-t A}\right\|_{\mathscr{L}\left(X_{0}\right)},\left\|t A e^{-t A}\right\|_{\mathscr{L}\left(X_{0}\right)}\right\} \leq M_{0} e^{-\omega t},
$$

for all $t \geq 0$. In this case, the real interpolation space $X_{1-1 / q, q}=\left(X, X_{1}\right)_{1-1 / q, q}$ has an equivalent norm (cf. [19], Section 5.1)

$$
\|x\|_{X_{1-1 / q, q}}=\left(\int_{0}^{1}\left\|A e^{-s A} x\right\|^{q}\right)^{1 / q} .
$$

It is well known that (cf. $[4,5,13]) A$ has the maximal $L^{q}$-regularity on the interval $I$ if and only if the singular integral operator $T$ defined through

$$
T f(t)=\int_{0}^{t} A e^{-(t-s) A} f(s) \mathrm{d} s, \quad f \in C_{0}^{\infty}(I, X)
$$

is well defined and can be extended onto $L^{q}(I, X)$ as a bounded linear operator.

As preparations for the discussions on the trace of the space $\mathbb{E}_{0}^{1, p(\cdot)}(I)$, let us recall the definition and construction of the abstract-valued function space of the range-varying type. For the detailed discussions, please refer to $[16,17]$.

Suppose that $\mathscr{A}$ is an ordered topological space with the order $\prec$, in which every order-bounded subset has the order supremum and order infimum. Suppose also $\mathscr{A}$ is totally orderbounded, i.e., there are $\alpha^{ \pm}$in another order space containing $\mathscr{A}$ such that $\alpha^{-} \prec \alpha \prec \alpha^{+}$for all $\alpha \in \mathscr{A}$. Under present situation, $\mathscr{A}$ is called a totally bounded lattice. Let $\left\{\alpha_{k}\right\} \subseteq \mathscr{A}$ and $\alpha \in \mathscr{A}$, we say that $\left\{\alpha_{k}\right\}$ is approaching $\alpha$, we mean that $\alpha_{k}<\beta$ for all $k \in \mathbb{N}$ and $\lim _{k \longrightarrow \infty} \alpha_{k}=\beta$ at the same time.

Let $\left\{X_{\alpha}: \alpha \in \mathscr{A}\right\}$ be a family of Banach spaces attached to $\mathscr{A}$. We say it is a regular Banach space net, provided the hypotheses are both fulfilled:

(1) If $\alpha<\beta$, then $X_{\beta} \hookrightarrow X_{\alpha}$, and there is a constant $C>0$ independent of $\alpha, \beta$ such that $\|x\|_{\alpha} \leq C\|x\|_{\beta}$ for all $x \in X_{\beta}$. 
(2) If $\left\{\alpha_{k}\right\}$ approaches $\beta$, then $\lim _{k \longrightarrow \infty}\|x\|_{\alpha_{k}}=\|x\|_{\beta}$ for all $x \in X_{\beta}$. Moreover, if $x \in X_{\alpha_{k}}$ for all $k \in \mathbb{N}$ and $C=\sup _{k \longrightarrow \infty}\|x\|_{\alpha_{i}}<\infty$, then $x \in X_{\beta}$ and $\|x\|_{\beta} \leq C$.

Let $I$ be an interval as above and $\Lambda(I)$ be the collection of all bounded subintervals of $I$. Consider the map $\theta: I \longrightarrow \mathscr{A}$. When we say $\theta$ is order-continuous, we mean that for any nest of intervals $\left\{J_{k} \in \Lambda(I): k=1,2, \ldots\right\}$ shrinking to $t$, the limit

$$
\lim _{k \longrightarrow \infty} \theta_{J_{k}}^{-}=\lim _{k \longrightarrow \infty} \theta_{J_{k}}^{+}=\theta(t)
$$

always holds, where $\theta_{J}^{-}$and $\theta_{J}^{+}$denote the order infimum and supremum of $\theta$ on $J$, respectively.

Define

$$
L^{0}\left(I, X_{\theta(\cdot)}\right)=\left\{f \in L^{0}(I, X):\left.f\right|_{J} \in L^{0}\left(J, X_{\theta_{J}^{-}}\right) \text {for all } J \in \Lambda(I) \text {, and } f(t) \in X_{\theta(t)} \text { for a.e. } t \in I\right\} \text {. }
$$

This is a linear space according to the addition and scalar multiplication of functions. Moreover, for all $f \in L^{0}\left(I, X_{\theta(.)}\right)$, the composite function $t \longmapsto\|f(t)\|_{\theta(t)}$ is measurable.
There are two types of range-varying function spaces derived from $L^{0}\left(I, X_{\theta(.)}\right)$, one is of continuous type defined through

$$
C^{-}\left(I, X_{\theta(\cdot)}\right)=\left\{f \in L^{0}\left(I, X_{\theta(\cdot)}\right):\left.f\right|_{J} \in C\left(J, X_{\theta_{J}^{-}}\right) \text {for all } J \in \Lambda(I) \text {, and } \sup _{t \in I}\|f(\mathrm{t})\|_{\theta(t)}<\infty\right\}
$$

which is a Banach space equipped with the norm $\sup _{t \in I}\|f(t)\|_{\theta(t)}$ or equivalently $\sup _{J \in \Lambda(I)}\left\|\left.f\right|_{J}\right\|_{C\left(J, X_{\theta^{-}}\right)}$. And the other is of an integral type defined through

$$
L^{p(\cdot)}\left(I, X_{\theta(\cdot)}\right)=\left\{f \in L^{0}\left(I, X_{\theta(\cdot)}\right):\|f(\cdot)\|_{\theta(\cdot)} \in L^{p(\cdot)}(I)\right\},
$$

with the Luxemburg norm

$$
\|f\|_{L^{p(\cdot)}\left(I, X_{\theta(\cdot)}\right)}=\inf \left\{\lambda>0: \int_{I}\left(\frac{\|f(t)\|_{\theta(t)}}{\lambda}\right)^{p(t)} \mathrm{d} t \leq 1\right\}
$$

where $p: I \longrightarrow[1, \infty)$ is a measurable variable exponent. If $\theta(t) \equiv 0$, then we obtain the familiar Lebesgue-Bochner space of variable exponent type $L^{p(\cdot)}\left(I, X_{0}\right)$.

Discussions in [16] tell us that, if we take $[0,1)$ as the totally bounded lattice, then $\left\{X_{\alpha}:=X_{\alpha, 1 /(1-\alpha)}: \alpha \in(0,1)\right.$, and $X_{0}=$ $X\}$ is a regular Banach space net. Hence, for the continuous exponent $p: I \longrightarrow(1, \infty)$, we obtain the linear space $L^{0}\left(I, X_{1-1 / p(\cdot), p(\cdot)}\right)$ and the Banach space $C^{-}\left(I, X_{1-1 / p(\cdot), p(\cdot)}\right)$. We can also construct the maximal regularity space with variable exponent $\mathbb{E}^{1, p(\cdot)}(I)=W^{1, p(\cdot)}(I, X) \cap L^{p^{(\cdot)}}\left(I, X_{1}\right)$ with the norm $\|u\|_{\mathbb{E}_{0}^{1, p(\cdot)}(I)}=\left\|u^{\prime}\right\|_{L^{p(\cdot)}(I, X)}+\|u\|_{L^{p(\cdot)}\left(I, X_{1}\right)}$ and homogeneous subspace $\mathbb{E}_{0}^{1, p(\cdot)}(I)=W_{0}^{1, p(\cdot)} \quad(I, X) \cap L^{p(\cdot)}$ $\left(I, X_{1}\right)$. All of them will be applied in the coming arguments.

\section{Main Results and Proofs}

We firstly focus on boundedness of the singular integral operator with operator-valued kernel on $L^{p(\cdot)}\left(\mathbb{R}^{N}, X\right)$.

Let $X$ and $Y$ be two Banach spaces, $\Upsilon=\{(x, y): x, y$ $\left.\in \mathbb{R}^{N}, x \neq y\right\}$, and let $k: \Upsilon \longrightarrow \mathscr{L}(Y, X)$ is a locally integrable function. Define a linear operator $T$ as follows:

$$
T f(x)=\int_{\mathbb{R}^{N}} k(x, y) f(y) \mathrm{d} y, \quad f \in C_{0}^{\infty}\left(\mathbb{R}^{N}, Y\right), x \bar{\epsilon} \operatorname{supp}(f) .
$$

$T$ is called a singular integral operator of strong $(q, q)$ type, provided it can be extended onto $L^{q}\left(\mathbb{R}^{N}, X\right)$ to $L^{q}\left(\mathbb{R}^{N}, Y\right)$ for some $1<q<\infty$, and there is a $C_{1}>0$ such that

$$
\|T f\|_{L^{q}\left(\mathbb{R}^{N}, X\right)} \leq C_{1}\|f\|_{L^{q}\left(\mathbb{R}^{N}, Y\right)},
$$

for all $f \in L^{q}\left(\mathbb{R}^{N}, X\right)$.

If there are constants $C_{2}>0, \delta>0$ such that

$$
\|k(x, y)\|_{\mathscr{L}(Y, X)} \leq \frac{C_{2}}{|x-y|^{N}}, \quad x \neq y,
$$

$$
\|k(x, y)-k(z, y)\|_{\mathscr{L}(Y, X)} \leq \frac{C_{2}|x-z|^{\delta}}{|x-y|^{-N-\delta}}, \quad|x-z| \leq \frac{|x-y|}{2},
$$

$$
\|k(y, x)-k(y, z)\|_{\mathscr{L}(Y, X)} \leq \frac{C_{2}|x-z|^{\delta}}{|x-y|^{-N-\delta}}, \quad|x-z| \leq \frac{|x-y|}{2},
$$

then $k$ is called a standard kernel. Here assumption (17) tells us that $k(x, y)$ is a singular kernel, and (18) and (19) together imply that $k(x, y)$ is locally Hölder continuous in some way. All of them are connected to the strong $(q, q)$ boundedness of $T$ in case that $k$ is a scalar kernel. And under the strong $(q, q)$ assumption of $T$, we only use (18) to deal with the strong $(p(\cdot), p(\cdot))$ property of $T$ for the operator-valued kernel.

We say $k$ satisfies the Hörmander's integral condition, if there is another constant $C_{3}>0$ such that for every cube $Q$ 
with sides parallel to the coordinate axes and all $y, z \in Q$, we have

$$
\int_{\mathbb{R}^{N} \backslash 2 Q}|k(x, y)-k(x, z)| \mathrm{d} x \leq C_{3},
$$

where $2 Q$ represents the cube with the same center and double sides of $Q$.

Similar to the scalar case, for the operator-valued kernel, we have [13].

Lemma 1. Under Hörmander's integral condition (20), a singular integral operator Tof strong $(q, q)$ type is also of weak $(1,1)$ type in the sense that

$$
m\left(\left\{x \in \mathbb{R}^{N}:\|T f(x)\|_{X}>\lambda\right\}\right) \leq \frac{C_{4}}{\lambda}\|f\|_{L^{1}\left(\mathbb{R}^{N}, Y\right)},
$$

for all $f \in L^{q}\left(\mathbb{R}^{N}, Y\right) \cap L^{1}\left(\mathbb{R}^{N}, Y\right)$ and some constant $C_{4}=C\left(N, q, C_{1}, C_{3}\right)>0$.

The following lemma is a natural extension of $[20,21]$ of the standard kernel from the scalar type to the operator- value type. For the convenience of the reader, we state it here and give it a complete proof.

Lemma 2. Let $\mathrm{T}$ be an operator defined through (15) with the standard kernel $k$. Suppose that T can be extended as a weak $(1,1)$ type operator as above and $0<s<1$, then for all $f \in C_{0}^{\infty}\left(\mathbb{R}^{N} ; Y\right)$, the scalar function $\|T f(\cdot)\|_{X}^{s}$ lies in the space $B M O\left(\mathbb{R}^{N}\right)$, and there is a constant $C_{5}=C\left(N, s, \delta, C_{4}\right)>0$ such that

$$
M^{\#}\left(\|T f\|_{X}^{s}\right)(x) \leq C_{5}(M f)^{s}(x)=: C_{5}\left(M\left(\|f\|_{Y}\right)\right)^{s}(x),
$$

for all $x \in \mathbb{R}^{N}$.

Proof. Take any $f \in C_{0}^{\infty}\left(\mathbb{R}^{N} ; Y\right)$ and $x_{0} \in \mathbb{R}^{N}$. Without loss of generality, assume that $M f\left(x_{0}\right)>0$. Let $Q$ be a cube containing $x_{0}$ with sides parallel to the coordinate axes. Consider the split $f=f_{1}+f_{2}, f_{1}=f \chi_{2 Q}$. For the first part $f_{1}$, we have

$$
\begin{aligned}
\frac{1}{|Q|} \int_{Q}\left\|T f_{1}(x)\right\|_{X}^{s} \mathrm{~d} x & =\frac{s}{|Q|} \int_{0}^{\infty} \lambda^{s-1} m\left(\left\{x \in Q:\left\|T f_{1}(x)\right\|_{X}>\lambda\right\}\right) \mathrm{d} \lambda \\
& \leq \frac{s}{|Q|}\left(\int_{0}^{t} \lambda^{s-1}|\mathrm{Q}| \mathrm{d} \lambda+\int_{t}^{\infty} \lambda^{s-1} \frac{C_{4}\left\|f_{1}\right\|_{L^{1}\left(\mathbb{R}^{N} ; Y\right)}}{\lambda} \mathrm{d} \lambda\right) \\
& =t^{s}+\frac{C_{4} t^{s-1}}{1-s} \frac{1}{|Q|} \int_{2 Q}\|f(y)\|_{Y} \mathrm{~d} y \\
& \leq t^{s}+\frac{2^{N} C_{4}}{1-s} M f\left(x_{0}\right) t^{s-1} .
\end{aligned}
$$

Take $t=M f\left(x_{0}\right)$, and we obtain

$$
\frac{1}{|Q|} \int_{Q}\left\|T f_{1}\right\|_{Y}^{s} \mathrm{~d} x \leq\left(1+\frac{2^{n} C_{1}}{1-s}\right)(M f)^{s}\left(x_{0}\right) \text {. }
$$

For the second part $f_{2}$, we have

$$
\begin{aligned}
& \frac{1}{|Q|} \int_{Q}\left|\left\|T f_{2}(x)\right\|_{X}-\left\|T f_{2}\left(x_{0}\right)\right\|_{X}\right|^{s} \mathrm{~d} x \\
& \leq\left(\frac{1}{|Q|} \int_{Q}\left\|T f_{2}(x)-T f_{2}\left(x_{0}\right)\right\|_{X} \mathrm{~d} x\right)^{s} .
\end{aligned}
$$

$$
\left\|T f_{2}(x)-T f_{2}\left(x_{0}\right)\right\|_{X} \leq \int_{\mathbb{R}^{N} \mid 2 Q}\left\|k(x, y)-k\left(x_{0}, y\right)\right\|_{\mathscr{L}(Y, X)}\|f(y)\|_{Y} \mathrm{~d} y
$$$$
\leq \sum_{j=1}^{\infty} \int_{2^{j+1} \mathrm{Q} \mid z^{2} \mathrm{Q}} \frac{\left|x-x_{0}\right|^{\delta}}{|x-y|^{N+\delta}}\|f(y)\|_{Y} \mathrm{~d} y
$$$$
\leq \sum_{j=1}^{\infty} 2^{(j-1) \delta} \int_{2^{j+1} Q \mid 2^{j} Q} \frac{\|f(y)\|_{Y}}{\left(2^{j} r\right)^{N}} \mathrm{~d} y
$$$$
\leq 2^{N} \sum_{j=1}^{\infty} 2^{(j-1) \delta} \cdot \frac{1}{\left|2^{j+1} Q\right|} \int_{2^{j+1} Q}\|f(y)\|_{Y} \mathrm{~d} y
$$$$
\leq \frac{2^{N}}{1-2^{\delta}} M f\left(x_{0}\right),
$$

Notice that for all $x \in Q$, by (18), 
where $r$ denotes the radius of $Q$, we obtain

$$
\frac{1}{|Q|} \int_{Q}\left|\left\|T f_{2}(x)\right\|_{X}-\left\|T f_{2}\left(x_{0}\right)\right\|_{X}\right|^{s} \mathrm{~d} x \leq\left(\frac{2^{N}}{1-2^{\delta}}\right)^{s}(M f)^{s}\left(x_{0}\right) \text {. }
$$

Putting the above two estimates together, we obtain

$$
\frac{1}{|Q|} \int_{Q}\left|\|T f(x)\|_{X}-\left\|T f_{2}\left(x_{0}\right)\right\|_{X}\right|^{s} \mathrm{~d} x \leq C(M f)^{s}\left(x_{0}\right) \text {, }
$$

for some constant $C_{5}=C\left(N, s, \delta, C_{4}\right)$, which means that $\|T f\|_{Y}^{s} \in \operatorname{BMO}\left(\mathbb{R}^{N}\right)$, and estimate (22) holds.

Given a variable exponent $p: \mathbb{R}^{N} \longrightarrow[1, \infty)$. We say $p$ is log-Hölder continuous, or symbolically $p \in \mathscr{P}^{\log }\left(\mathbb{R}^{N}\right)$, if there are constants $C_{0}>0$ and $p_{\infty} \geq 1$ such that

$$
\begin{aligned}
& |p(x)-p(y)| \leq \frac{C_{0}}{\log \left(e+|x-y|^{-1}\right)}, \\
& \left|p(x)-p_{\infty}\right| \leq \frac{C_{0}}{\log (e+|x|)},
\end{aligned}
$$

for all $x, y \in \mathbb{R}^{N}$.

Remark 1. If $\Omega$ is a bounded domain of $\mathbb{R}^{N}$, then $p$ is logHölder continuous on $\Omega$, if and only if the first inequality of (29) is satisfied.

Next lemma is an important result in harmonic analysis, and it was first proved in [14] for bounded exponents and later extended to general cases in some literatures. For the complete proof with detail discussions, please refer to [15].

Lemma 3. Assume that $\mathscr{P}^{\log }\left(\mathbb{R}^{N}\right)$ and $p^{-}>1$, then the maximal operator $M$ is bounded from $L^{p(\cdot)}\left(\mathbb{R}^{N}\right)$ to $L^{p(\cdot)}\left(\mathbb{R}^{N}\right)$, i.e., there is a constant $C_{6}=C\left(N, p^{-}, C_{0}\right)>0$ such that

$$
\|M f\|_{L^{p(\cdot)}\left(\mathbb{R}^{N}\right)} \leq C_{6}\|f\|_{L^{p(\cdot)}\left(\mathbb{R}^{N}\right)} .
$$

Furthermore, under the extra assumption $p^{+}<\infty$, for the sharp operator $M^{\#}$, there is another constant $C_{7}=C\left(p^{ \pm}, C_{6}\right)>0$ for which estimate

$$
\|f\|_{L^{q}\left(\mathbb{R}^{N}\right)} \leq C_{7}\left\|M^{\#} f\right\|_{L^{q}\left(\mathbb{R}^{N}\right)},
$$

for all $f \in L^{q}\left(\mathbb{R}^{N}\right)$ (refer to [22], P.148).

Putting all the facts together, we obtain the following.

Theorem 1. Let $T$ be a singular integral operator of strong $(q, q)$ type for some $1<q<\infty$ with the standard kernel $k$ satisfying Hörmander's integral condition and $p \in \mathscr{P}^{\log }\left(\mathbb{R}^{N}\right)$ be a variable exponent satisfying $1<p^{-} \leq p^{+}<\infty$. Then, $T$ is bounded from $L^{p(\cdot)}\left(\mathbb{R}^{N} ; Y\right)$ to $L^{p(\cdot)}\left(\mathbb{R}^{N} ; X\right)$ with the bounds $C_{8}=C\left(N, p^{ \pm}, \delta, C_{1}, C_{2}, C_{3}\right)>0$.

Proof. Take a constant exponent $s$ such that $0<s<1$, and then the variable exponent $p(\cdot) / s$ is also log-Hölder continuous the same constant $C_{0}$, and $(p(\cdot) / s)^{-}=p^{-} / s>1$ and $(p(\cdot) / s)^{+}=p^{+} / s<\infty$. Thus, combining (22), (30), and (31), we can deduce that

$$
\begin{aligned}
\|T f\|_{L^{p(\cdot)}\left(\mathbb{R}^{N} ; X\right)} & =\|\| T f\left\|_{X}^{s}\right\|_{L^{p(\cdot) / s}\left(\mathbb{R}^{N}\right)}^{1 / s} \\
& \leq C_{7}\left\|M^{\#}\left(\|T f\|_{X}^{s}\right)\right\|_{L^{p(\cdot) / s}\left(\mathbb{R}^{N}\right)}^{1 / s} \\
& \leq C_{5} C_{7}\left\|M^{s}\left(\|f\|_{Y}\right)\right\|_{L^{p(\cdot) / s}}^{1 / s}\left(\mathbb{R}^{N}\right) \\
& =C_{5} C_{7}\|M f\|_{L^{p(\cdot)}}\left(\mathbb{R}^{N} ; Y\right) \\
& \leq C_{8}\|f\|_{L^{p(\cdot)}\left(\mathbb{R}^{N} ; Y\right)},
\end{aligned}
$$

where the constant $C_{8}=C_{6} C_{5} C_{7}=C\left(N, p^{ \pm}, \delta, C_{1}\right.$, $\left.C_{2}, C_{3}\right)$.

Remark 2. This conclusion is a natural extension of that in [15], Section 1.6.3 for the singular integral operator from the scalar type to the abstract-valued type. For another treatment of the extension, please refer to [23].

Now we can establish the maximal $L^{p(\cdot)}$-regularity for the sectorial operator $A$. Define

$$
K(t)= \begin{cases}A e^{-t A}, & \text { if } t>0 \\ 0, & \text { if } t \leq 0\end{cases}
$$

Straight calculations show that $k(t, s)=K(t-s)$ is a standard kernel satisfying Hörmander's integral condition, and $T f$ can be expressed by $K * \widetilde{f}$, where $\widetilde{f}$

$$
\tilde{f}(t)= \begin{cases}f(t), & \text { if } t \in I, \\ 0, & \text { if } t \in \mathbb{R} \backslash I,\end{cases}
$$

is the zero extension of $f$. In this setting, $A \in M \mathscr{R}_{q}(I)$ is equivalent to say that $T$ is a singular integral operator of strong $(q, q)$ type.

Given a variable exponent $p \in \mathscr{P}(I)$ satisfying the logHölder condition (29) with $\mathbb{R}^{N}$ replaced by $I$ and $1<p^{-} \leq p^{+}<\infty$. From [15], Section 4.1, we know that $p$ has an extension $\tilde{p} \in \mathscr{P}(\mathbb{R})$ with the same constant $C_{0}$ and $\widetilde{p}^{ \pm}=p^{ \pm}$. Analogous to $\mathbb{E}^{1, q}(I)$ and $\mathbb{E}_{0}^{1, q}(I)$, define the maximal $L^{p(\cdot)}$-regularity space $\mathbb{E}^{1, p(\cdot)}(I)$ and its closed subspace $\mathbb{E}_{0}^{1, p(\cdot)}(I)$ with the norm

$$
\|u\|_{\mathbb{E}^{1, p(\cdot)}(I)}=\|u\|_{W^{1, p(\cdot)}(I, X)}+\|u\|_{L^{p(\cdot)}\left(I, X_{1}\right)} .
$$

Applying Theorem 1, we can drive the following.

Theorem 2. Assume that $A \in \mathscr{M} \mathscr{R}_{q}(I)$ for some $1<q<\infty$ and $p \in \mathscr{P}(I)$ with $1<p^{-} \leq p^{+}<\infty$. Then, A satisfies the maximal $L^{p^{(\cdot)}}$-regularity on $I$, that is, for all $f \in L^{p(\cdot)}(I, X)$, there is a unique function $u \in \mathbb{E}_{1, p(\cdot)}(I)$ solving (3) with $u_{0}=0$, and satisfying

$$
\|u\|_{\mathbb{E}^{1, p(\cdot)}(I)} \leq C\|f\|_{L^{p(\cdot)}\left(I, X_{1}\right)},
$$

with the constant $C>0$ depending on $A, C_{0}, C_{q}$, and $p^{ \pm}$.

In the following paragraphs, we turn our attention to the trace of $\mathbb{E}^{1, p(\cdot)}(I)$. Here and after we need assumption (7) for the semigroup $e^{-t A}$. Denote by $\gamma \mathbb{E}^{1, p(\cdot)}(I)$ the trace space of $\mathbb{E}^{1, p(\cdot)}(I)$, that is, 
$\gamma \mathbb{E}^{1, p(\cdot)}(I)=\left\{u_{0} \in X: \exists u \in \mathbb{E}^{1, p(\cdot)}(I)\right.$ s.t. $\left.u(0)=u_{0}\right\}$,

with the norm

$$
\left\|u_{0}\right\|_{\mathbb{E}^{1, p(\cdot)}(I)}=\inf \left\{\|u\|_{\mathbb{E}^{1, p(\cdot)}(I)}: u \in \mathbb{E}^{1, p(\cdot)}(I), u(0)=u_{0}\right\} .
$$

Proposition 1. Suppose that $p \in \mathscr{P}^{\log }\left(\mathbb{R}^{+}\right)$with $1<p^{-} \leq p^{+}$ $<\infty$, then

$$
\gamma \mathbb{E}^{1, p(\cdot)}\left(\mathbb{R}^{+}\right)=X_{1-1 / p(0), p(0)},
$$

with the equivalent norms.

Proof. Firstly, for all $u_{0} \in X_{1-1 / p(0), p(0)}$ with $\left\|u_{0}\right\|_{X_{1-1 / p(0), p(0)}}$ $\leq 1$, we have $\left\|u_{0}\right\|_{X} \leq C$ for some constant $C>0$ independent of $u_{0}$, and $\int_{0}^{1}\left\|e^{-t A} u_{0}\right\|^{p(0)} \mathrm{d} t \leq 1$. Consider the split

$$
\int_{0}^{\infty}\left\|A e^{-t A} u_{0}\right\|^{p(t)} \mathrm{d} t=\left(\int_{0}^{1}+\int_{1}^{\infty}\right)\left\|A e^{-t A} u_{0}\right\|^{p(t)} \mathrm{d} t:=I_{1}+I_{2} .
$$

For the second part, we have

$$
I_{2} \leq \int_{1}^{\infty}\left(\frac{M_{0}}{t} e^{-\omega t}\left\|u_{0}\right\|_{X}\right)^{p(t)} \mathrm{d} t \leq \frac{1}{\omega p^{-}} \max \left\{\left(M_{0} C\right)^{p^{+}}, 1\right\} .
$$

Define $E=\{t \in[0,1]: p(t) \leq p(0)\}$, let $p_{1}(t)=p(t) \chi_{E}+$ $p(0)\left(1-\chi_{E}\right)$ and $p_{2}(t)=p(t)\left(1-\chi_{E}\right)+p(0) \chi_{E}$, then both of $p_{1}$ and $p_{2}$ are log-Hölder continuous with the same constant $C_{0}$. Thus, for the first part $I_{1}$, we have

$$
I_{1} \leq \int_{0}^{1}\left\|A e^{-t A} u_{0}\right\|^{p_{1}(t)} \mathrm{d} t+\int_{0}^{1}\left\|A e^{-t A} u_{0}\right\|^{p_{2}(t)} \mathrm{d} t:=I_{1,1}+I_{1,2}
$$

where

$$
\begin{aligned}
I_{1,1} & \leq \int_{0}^{1}\left\|A e^{-t A} u_{0}\right\|^{p(0)} \mathrm{d} t+\left(1-\frac{p^{-}}{p(0)}\right) \leq 2, \\
I_{1,2} & \leq \int_{0}^{1}\left\|A e^{-t A} u_{0}\right\|^{p(0)}\left(\frac{M\left\|u_{0}\right\|_{X}}{t}\right)^{p_{2}(t)-p(0)} \mathrm{d} t \\
& \leq \max \left\{\left(M_{0} C\right)^{p^{+}-p^{-}}, 1\right\} \int_{0}^{1}\left\|A e^{-t A} u_{0}\right\|^{p(0)} t^{-\left(C_{0} / \log (e+1 / t)\right)} \mathrm{d} t \\
& \leq \max \left\{\left(M_{0} C\right)^{p^{+}-p^{-}}, 1\right\} e^{C_{0}} .
\end{aligned}
$$

Putting all the parts together, we obtain $\int_{0}^{\infty}\left\|e^{-t A} u_{0}\right\|^{p(t)} \mathrm{d} t \leq C$ with the constant $C>0$ independent of $u_{0}$. And by scaling arguments, we have $\left\|e^{-t A} u_{0}\right\|_{\mathbb{E}^{1, p(\cdot)}\left(\mathbb{R}^{+}\right)}$ $\leq C\left\|u_{0}\right\|_{X_{1-1 / p(0), p(0)}}$, which in turn yields $\left\|u_{0}\right\|_{\gamma \mathbb{E}^{1, p(\cdot)}\left(\mathbb{R}^{+}\right)} \leq$ $C\left\|u_{0}\right\|_{X_{1-1 / p(0)}(0)}$.

Conversely, suppose that $u \in \mathbb{E}^{1, p(\cdot)}\left(\mathbb{R}^{+}\right)$with $\|u\|_{\mathbb{E}^{1, p(\cdot)}\left(\mathbb{R}^{+}\right)} \leq 1$ and $u(0)=u_{0}$. By the unit ball property, we have $\int_{0}^{1}\|A u(t)\|^{p(t)} \mathrm{d} t \leq 1$ and $\left\|u^{\prime}\right\|_{L^{p(\cdot)}([01], X)} \leq 1$. By imbedding $W^{1, p(\cdot)}\left(\mathbb{R}^{+}, X\right) \hookrightarrow C([0,1], X)$, we get the estimate $\|u\|_{C([0,1], X)} \leq C$ for some constant $C>0$ independent of $u$. Notice that

$$
u_{0}=u(t)-\int_{0}^{t} u^{\prime}(s) \mathrm{d} s
$$

we have

$$
\begin{aligned}
\left\|A e^{-t A} u_{0}\right\|_{L^{p(0)}([0,1], X)} \leq & \left\|A e^{-t A} u\right\|_{L^{p(0)}([0,1], X)} \\
& +\left\|A e^{-t A} \int_{0}^{t} u^{\prime}(s) \mathrm{d} s\right\|_{L^{p(0)}([0,1], X)} .
\end{aligned}
$$

Since

$$
\begin{aligned}
\int_{0}^{1}\left\|A e^{-t A} u(t)\right\|^{p(0)} \mathrm{d} t & \\
\leq & M_{0}^{p(0)} \int_{E}\|A u(t)\|^{p(t)}\left\|\frac{u(t)}{t}\right\|^{p^{(0)-p(t)}} \mathrm{d} t \\
& +M_{0}^{p(0)} \int_{[0,1] \backslash E}\|A u(t)\|^{p(0)} \mathrm{d} t \\
\leq & M_{0}^{p(0)} \max \left\{1,\|u\|_{C([0,1], X)}\right\}^{p(0)-p^{-}} \\
& \quad \int_{0}^{1}\|A u(t)\|^{p(t)} t^{-\left(C_{0} / \log (e+1 / t)\right)} \mathrm{d} t \\
& +M_{0}^{p(0)}\left(\int_{0}^{1}\|A u(t)\|^{p^{(t)}} \mathrm{d} t+1\right) \\
\leq & M_{0}^{p^{+}}\left(e^{C_{0}} \max \{1, C\}^{p^{+}-p^{-}}+2\right), \\
\int_{0}^{1} \| & A e^{-t A} \int_{0}^{t} u^{\prime}(s) \mathrm{d} s \|^{p(0)} \mathrm{d} t \\
\leq & M_{0}^{p^{+}}\left[\left(\max \{1,2 C\}^{p^{+}-p^{-}} e^{C_{0}}+1\right) \max \left\{C_{6}^{p^{+}}, C_{6}^{p^{-}}\right\}+1\right] \\
\leq & M_{0}^{p(0)} \int_{E}\left\|\frac{1}{t} \int_{0}^{t} u^{\prime}(s) \mathrm{d} s\right\|^{p(t)}\|u(t)-u(0)\|^{p(0)-p(t)} \mathrm{d} t \\
& +M_{0}^{p(0)} \int_{[0,1] \backslash E}^{p(0)} \max \left\{1,2\left\|\int_{0}^{t} \int_{0}^{t} u^{\prime}(s) \mathrm{d} s\right\|_{C([0,1], X)} \|^{p(0)} \mathrm{d} t\right.
\end{aligned}
$$


where

$$
\begin{aligned}
\int_{0}^{1}\left(M\left(\left\|u^{\prime}\right\|\right)(t)\right)^{p^{(t)} \mathrm{d} t} & \leq \max \left\{\left\|M\left(\left\|u^{\prime}\right\|\right)\right\|_{L^{p())}[0,1]^{\prime}}^{p^{+}}\left\|M\left(\left\|u^{\prime}\right\|\right)\right\|_{L^{p()}[0,1]}^{p^{-}}\right\} \\
& \leq \max \left\{\left(C_{6}\left\|u^{\prime}\right\|_{L^{p())}[0,1]}\right)^{p^{+}},\left(C_{6}\left\|u^{\prime}\right\|_{L^{p^{(\cdot)}}[0,1]}\right)^{p^{-}}\right\} \\
& \leq \max \left\{C_{6}^{p^{+}}, C_{6}^{p^{-}}\right\},
\end{aligned}
$$

by the unit ball property of $L^{p(\cdot)}$ and boundedness of the maximal operator, we then have $\int_{0}^{1}\left\|A e^{-t A} u_{0}\right\|^{p(0)} \mathrm{d} t \leq C$ with the constant $C>0$ independent of $u$. By the scaling arguments and definition of $\gamma \mathbb{E}^{1, p(\cdot)}\left(\mathbb{R}^{+}\right)$, we can also derive that $\left\|u_{0}\right\|_{X_{1-1 / p(0), p(0)}} \leq C\left\|u_{0}\right\|_{\mathbb{E}^{1, p(\cdot)}\left(\mathbb{R}^{+}\right)}$. Thus, equivalence of $\gamma \mathbb{E}^{1, p(\cdot)}\left(\mathbb{R}^{+}\right)$and $X_{1-1 / p(0), p(0)}$ has been reached.

Proposition 2. Under the same assumptions upon $p$ as above, we have

$$
\mathbb{E}^{1, p(\cdot)}\left(\mathbb{R}^{+}\right) \hookrightarrow C^{-}\left(\mathbb{R}^{+}, X_{1-1 / p(\cdot), p(\cdot)}\right) .
$$

Proof. irstly, for every bounded subinterval $J$ of $\mathbb{R}^{+}$, imbedding

$$
\mathbb{E}^{1, p(\cdot)}(J) \hookrightarrow \mathbb{E}^{1, p_{J}^{-}}(J) \hookrightarrow C\left(J, X_{1-1 / p_{J}^{-}, p_{J}^{-}}\right),
$$

holds with the imbedding bounds depending on $J$, from which we obtain

$$
\mathbb{E}^{1, p(\cdot+s)}\left(\mathbb{R}^{+}\right) \subseteq L_{-}^{0}\left(\mathbb{R}^{+}, X_{1-1 / p(\cdot), p(\cdot)}\right),
$$

where the range-varying function space $L_{-}^{0}\left(\mathbb{R}^{+}, X_{1-1 / p(\cdot), p(\cdot)}\right)$ comes from [17].

For each $s>0$, consider the translation operator $(T(s) u)(t)=u(t+s)$. Obviously, $T(s)$ is a bounded linear operator from $\mathbb{E}^{1, p(\cdot)}\left(\mathbb{R}^{+}\right)$to $\mathbb{E}^{1, p(+s)}\left(\mathbb{R}^{+}\right)$, and

$$
\|T(s) u\|_{\mathbb{E}^{1, p(++s)}\left(\mathbb{R}^{+}\right)} \leq\|u\|_{\mathbb{E}^{1, p(\cdot)}\left(\mathbb{R}^{+}\right)} .
$$

Another fact is that for every $s>0$, the translated exponent $T(s) p=p(\cdot+s)$ is also log-Hölder continuous with the same constant $C_{0}$ and $1<p^{-} \leq(T(s) p)^{-} \leq(T(s) p)^{+} \leq p^{+}<\infty$. Thus, for all $u \in \mathbb{E}^{1, p(\cdot)}\left(\mathbb{R}^{+}\right)$and all $s>0$, using Proposition 1 , we have

$$
\begin{array}{r}
\|u(s)\|_{X_{1-1 / p(s), p(s)}} \leq C\|(T(s) u)(0)\|_{\gamma \mathbb{E}^{1, p(++s)}\left(\mathbb{R}^{+}\right)} \\
\leq C\|T(s) u\|_{\mathbb{E}^{1, p(+s)}\left(\mathbb{R}^{+}\right)} \leq C\|u\|_{\mathbb{E}^{1, p(\cdot)}\left(\mathbb{R}^{+}\right) .} .
\end{array}
$$

Therefore, $u \in C^{-}\left(\mathbb{R}^{+}, X_{1-1 / p(\cdot), p(\cdot)}\right)$ and

$$
\|u\|_{C^{-}\left(\mathbb{R}^{+}, X_{1-1 / p(\cdot), P(\cdot)}\right)} \leq C\|u\|_{\mathbb{E}^{1, p(\cdot)}\left(\mathbb{R}^{+}\right)},
$$

with a constant $C>0$ independent of $u$ (refer to [17]).

Theorem 3. Suppose that $p \in \mathscr{P}^{\log }([0, b])$ with $1<p^{-} \leq p^{+}$ $<\infty$, then

$$
\mathbb{E}_{0}^{1, p(\cdot)}([0, b]) \hookrightarrow C^{-}\left([0, b], X_{1-1 / p(\cdot), p(\cdot)}\right),
$$

with the imbedding bounds independent of $T>0$.

Proof. Let

$$
\begin{aligned}
& \widetilde{p}(t)= \begin{cases}p(t), & 0 \leq t \leq b, \\
p(2 b-t), & b \leq t \leq 2 b, \\
p(0), & t \geq 2 b,\end{cases} \\
& \widetilde{u}(t)= \begin{cases}u(t), & 0 \leq t \leq b, \\
u(2 b-t), & b \leq t \leq 2 b, \\
0, & t \geq 2 b .\end{cases}
\end{aligned}
$$

It is easy to check that $\tilde{p} \in \mathscr{P}^{\log }\left(\mathbb{R}^{+}\right)$with $\widetilde{p}^{ \pm}=p^{ \pm}$and $\widetilde{p}_{\infty}=p(0), \widetilde{u} \in \mathbb{E}_{0}^{1, p(\cdot)}\left(\mathbb{R}^{+}\right)$, and

$$
\|\widetilde{u}\|_{\mathbb{E}_{0}^{\left(, \tilde{p}^{(\cdot)}\left(\mathbb{R}^{+}\right)\right.}} \leq 2\|u\|_{\mathbb{E}_{0}^{1, p(\cdot)}([0, b])} .
$$

By invoking Proposition 2, we know that $\tilde{u} \in C^{-}\left(\mathbb{R}^{+}, X_{1-1 / \widetilde{p}(\cdot), \widetilde{p}(\cdot)}\right)$, and

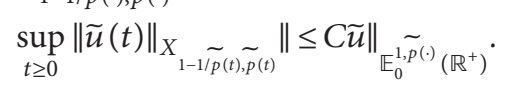

Notice that $\widetilde{p}(t)=p(t)$ and $\widetilde{u}(t)=u(t)$ for all $t \in[0, b]$, we obtain

$$
\sup _{t \in[0, b]}\|u(t)\|_{X_{1-1 / p(t), p(t)}} \leq C\|\widetilde{u}\|_{\mathbb{E}_{0}^{1, p(\cdot)}\left(\mathbb{R}^{+}\right)} \leq 2 C\|u\|_{\mathbb{E}_{0}^{1, p(\cdot)}([0, b])} .
$$

Thus, the proof has been proved.

At the end of the paper, we will use the maximal $L^{p(\cdot)}$-regularity results to deal with the semilinear evolution equation:

$$
\left\{\begin{array}{l}
u^{\prime}(t)+A u=F(t, u), \quad t>0, \\
u(0)=u_{0},
\end{array}\right.
$$

where $A \in \mathscr{M}_{\mathscr{R}_{q}}\left(\mathbb{R}^{+}\right)$for some $1<q<\infty, 0<b_{0} \leq \infty$, $p \in \mathscr{P}^{\log }\left[0, b_{0}\right]$, and $F:\left[0, b_{0}\right) \times X \longrightarrow X$ is a nonlinear map fulfilling assumptions $H(F)$ as follows:

(1) For almost all $t \in\left[0, b_{0}\right), F(t, \cdot)$ can be defined and locally Lipschitz continuous on $X_{1-1 / p(t), 1 / p(t)}$

(2) For all $u \in L^{0}\left(0, b_{0} ; X_{1-1 / p(\cdot), 1 / p(\cdot)}\right)$, the compound function $t \longrightarrow F(t, u(t))$ is strongly measurable on $\left[0, b_{0}\right)$

(3) $u_{0} \in X_{1-1 / p(0), 1 / p(0)}$, and $F\left(\cdot, e^{-\cdot A} u_{0}\right) \in L^{p(\cdot)}\left(0, b_{0}\right.$; $X)$ 
Theorem 4. Under present situations, for every $r>0$, there is a number $0<b<b_{0}$, such that for each $u_{1} \in \mathscr{B}\left(u_{0}, r\right)$, and semilinear equation (59) has a unique solution $u(\cdot)=u\left(\cdot, u_{1}\right) \in \mathbb{E}_{1, p(\cdot)}(I) \cap C^{-}\left(I, X_{1-1 / p(\cdot), p(\cdot)}\right)$ on the interval $I=[0, b]$ with the initial condition $u(0)=u_{1}$. Here,

$$
\mathscr{B}\left(u_{0}, r\right)=\left\{u \in X_{1-1 / p(0), p(0)}:\left\|u-u_{0}\right\|_{X_{1-1 / p(0), p(0)}}<r\right\},
$$

is a ball in $X_{1-1 / p(0), p(0)}$. Moreover, for every two points $u_{i} \in \mathscr{B}\left(u_{0}, r\right), i=1,2$, the corresponding solutions $u\left(\cdot, u_{i}\right)$, $i=1,2$, satisfy

$$
\begin{aligned}
& \max \left\{\left\|u\left(\cdot, u_{1}\right)-u\left(\cdot, u_{2}\right)\right\|_{C^{-}(I, 1-1 / p(\cdot), p(\cdot))}\right. \\
& \left.\left\|u\left(\cdot, u_{1}\right)-u\left(\cdot, u_{2}\right)\right\|_{\mathbb{E}_{1, p(\cdot)}(I)}\right\} \\
& \leq C\left\|u_{1}-u_{2}\right\|_{1-1 / p(0), p(0)} .
\end{aligned}
$$

Proof of this theorem is much similar to that completed in [16] where embedding ${ }_{0} \mathbb{E}_{1, p^{+}}(I) \hookrightarrow C^{-}\left(I, X_{1-1 / p(\cdot), p(\cdot)}\right)$ is replaced by ${ }_{0} \mathbb{E}_{1, p(\cdot)}(I) \hookrightarrow C^{-}\left(I, X_{1-1 / p(\cdot), p(\cdot)}\right)$.

Remark 3. Using the maximal $L^{p(\cdot)}$-regularity theory, hypothesis $F\left(\cdot, e^{-\cdot A} u_{0}\right) \in L^{p^{+}}\left(0, b_{0} ; X\right)$ used in [16] is dropped. Instead, here, a weaker assumption $H(F)(3)$ : $F\left(\cdot, e^{-\cdot A} u_{0}\right) \in L^{p(\cdot)}\left(0, b_{0} ; X\right)$ is applied. In this sense, Theorem 4 is an improvement of that in [16].

\section{Conclusions and Discussion}

In this paper, we study the maximal $L^{p(\cdot)}$-regularity for the sectorial operators. By extending the boundedness of singular integral operators from the scalar type to abstractvalued type; we see that, if a sectorial operator $A$ lies in $\mathscr{M} \mathscr{R}_{q}(I)$ for some $1<q<\infty$, then it lies in $\mathscr{M}_{\mathscr{R}_{p(\cdot)}}(I)$ for every Hölder continuous variable exponent $p(\cdot)$ with $1<p^{-}<p^{+}<\infty$. We also prove that if $-A$ generates an exponentially decaying analytic semigroup, then for the maximal regular space $\mathbb{E}_{1, p(\cdot)}\left(\mathbb{R}^{+}\right)$, its trace space is exactly $X_{1-1 / p(0), p(0)}$, and the homogeneous maximal regular space ${ }_{0} \mathbb{E}_{1, p(\cdot)}(I)$ can be embedded continuously into the rangevarying function space $C^{-}\left(I, X_{1-1 / p(\cdot), p(\cdot)}\right)$ with the embedding bounds independent of the length of the interval $I$. Different to the constant exponent type, translation series $\left\{T_{s}: L^{p(\cdot)}\left(\mathbb{R}^{+}, X\right) \longrightarrow L^{p(\cdot+s)}\left(\mathbb{R}^{+}, X\right), s \geq 0\right\}$ could not make up a $C_{0}$ semigroup on $L^{p(\cdot)}\left(\mathbb{R}^{+}, X\right)$, since $L^{p(\cdot)}\left(\mathbb{R}^{+}, X\right)$ does not have the translation-invariant property. Consequently, whether or not the following estimates:

$$
\begin{aligned}
& \sup _{\lambda \geq 0}\left\|\lambda\left(\lambda+\partial_{t}+A\right)^{-1}\right\|_{\mathscr{L}\left(L^{p(\cdot)}(I, X), L^{p(\cdot)}(I, X)\right)}<\infty, \\
& \sup _{\lambda \geq 0}\left\|\left(\lambda+\partial_{t}+A\right)^{-1}\right\|_{\mathscr{L}\left(L^{p(\cdot)}(I, X)_{, 0} \mathbb{E}_{1, p(\cdot)}(I)\right)}<\infty,
\end{aligned}
$$

still hold for the variable exponents remains unknown. We also wonder that under what situations maximal $L^{p(\cdot)}$-regularity can be preserved under time-dependent perturbation $B(t)$.

\section{Data Availability}

The data used to support the findings of this study are available from the corresponding author upon request.

\section{Conflicts of Interest}

The authors declare that there are no conflicts of interest regarding the publication of this paper.

\section{Acknowledgments}

This work was supported by the National Natural Science Foundation of China (11771223) and Preresearch Project of Nantong University (17ZY01).

\section{References}

[1] A. Pazy, Semigroup of Linear Operators and Applications to Partial Differential Equations, Springer-Verlag, New York, USA, 1983.

[2] A. Benedek, A. P. Calderon, and R. Panzone, "Convolution operators on Banach space valued functions," Proceedings of the National Academy of Sciences, vol. 48, no. 3, pp. 356-365, 1962.

[3] M. Hieber, "Operator-valued fourier multipliers," in Topics in Nonlinear Analysis, J. Escher and G. Simonnet, Eds., Birkhäuser, Basel, Switzerland, pp. 363-380, 1999.

[4] G. Dore, " $L_{p}$ regularity for abstract differential equations," Lecture Notes in Mathematics, Springer-Verlag, vol. 1540, no. 1, pp. 25-38, Berlin, Germany, 1993.

[5] J. Prüss, "Maximal regularity for evolution equations in $L_{p^{-}}$ spaces," Conferenze del Seminario di Matematica dell'Universitá di Bari, vol. 285, pp. 1-39, 2002.

[6] Y. Giga and H. Sohr, "On the Stokes operator in exterior domains," Journal of the Faculty of Science, the University of Tokyo Sec. IA, vol. 36, pp. 103-130, 1989.

[7] Y. Giga and H. Sohr, "Abstract $L_{p}$ estimates for the Cauchy problem with applications to the Navier-Stokes equations in exterior domains," Journal of Functional Analysis, vol. 102, no. 1, pp. 72-94, 1991.

[8] M. Hieber and J. Prüss, "Heat kernels and maximal $L_{p^{-}} L_{q^{-}}$ estimates for parabolic evolution equations," Communications in Partial Differential Equations, vol. 22, pp. 1647-1669, 1998.

[9] P. C. Kunstmann, "Maximal $L_{p}$ regularity for second order elliptic operators with uniformly continuous coefficients on domains," Evolution Equations: Applications to Physics, Industry, Life Sciences and Economics, vol. 55, pp. 293-305, 2003.

[10] J. Prüss and G. Simonett, "Maximal regularity for evolution equations in weighted $L_{p}$-spaces," Archiv der Mathematik, vol. 82, no. 5, pp. 415-431, 2004.

[11] M. Köhne, J. Prüss, and M. Wilke, "On quasilinear parabolic evolution equations in weighted $L_{p}$-spaces," Journal of Evolution Equations, vol. 10, no. 2, pp. 443-463, 2010.

[12] J. LeCrone, J. Prüss, and M. Wilke, "On quasilinear parabolic evolution equations in weighted $L_{p}$-spaces II," Journal of Evolution Equations, vol. 14, no. 3, pp. 509-533, 2014.

[13] R. Chill and A. Fiorenza, "Singular integral operators with operator-valued kernels, and extrapolation of maximal regularity into rearrangement invariant Banach function spaces," Journal of Evolution Equations, vol. 14, no. 4-5, pp. 795-828, 2014. 
[14] L. Diening, "Maximal function on generalized Lebesgue spaces $L^{p(\cdot)}$," Mathematical Inequalities \& Applications, vol. 7, no. 2, pp. 245-253, 2004.

[15] L. Diening, P. Harjulehto, P. Hästö, and M. Rủžička, "Lebesgue and Sobolev spaces with variable exponents," Lecture Notes in Mathematics, vol. 2017, Springer-Verlag, Berlin, Germany, 2011.

[16] Q. Zhang and G. Li, "On the $X(\cdot)$-valued function space: definition, property and applications," Journal of Mathematical Analysis and Applications, vol. 440, no. 1, pp. 48-64, 2016.

[17] Q. Zhang and G. Li, "Classification and geometrical properties of the $X_{\theta(\cdot)}$ - valued function spaces," Journal of Mathematical Analysis and Applications, vol. 452, no. 1, pp. 1359-1387, 2017.

[18] H. Amann, Linear and Quasilinear Parabolic Problems, Vol. 1, Abstract Linear Theory, Birkhäuser, Basel, Switzerland, 1995.

[19] A. Lunardi, Interpolation Theory, Vol. 9, Scuola Normale Superiore di Pisa (Nuova Series), Florence, Italy, 2nd edition, 2009.

[20] J. Álvarez and C. Pérez, "Estimates with $A_{\infty}$ weights for various singular integral operators," Bollettino dell'Unione Matematica Italiana, vol. 8, no. 7, pp. 123-133, 1994.

[21] M. Lorente, M. S. Riveros, and A. de la Torre, "Weighted estimates for singular integral operators satisfying Hörmander's conditions of young type," Journal of Fourier Analysis and Applications, vol. 11, no. 5, pp. 457-509, 2005.

[22] E. Stein, "Harmonic analysis: real-variable methods, orthogonality, and oscillatory integrals," Volume 43 of Princeton Mathematical Series (With the Assistance of Timothy S. Murphy, Monographs in Harmonic Analysis, III), Princeton University Press, Princeton, NJ, USA, 1993.

[23] C. Shen and J. Xu, "Caldeón-Zygmund operators on Banach space valued Lebesgue spaces with variable exponent," $A d$ vance in Mathematics (In Chinese), vol. 46, no. 3, pp. 441-452, 2017. 